\title{
CXCL10 enhances radiotherapy effects in HeLa cells through cell cycle redistribution
}

\author{
LING-LIN YANG, BI-QIONG WANG, LAN-LAN CHEN, HUI-QUN LUO and JING-BO WU
}

Affiliated Hospital of Luzhou Medical College, Luzhou, Sichuan 646000, P.R. China

Received June 6, 2011; Accepted October 11, 2011

DOI: $10.3892 / \mathrm{ol} .2011 .472$

\begin{abstract}
Radiotherapy is a crucial treatment for cervical cancer, the second most common type of cancer in women worldwide. In this study, we investigated the effects of CXC chemokine ligand 10 (CXCL10) gene therapy combined with radiotherapy on cervical cancer using HeLa cells. TUNEL assay revealed that the apoptotic rate in the combined treatment of CXCL10 gene therapy and radiotherapy was greatly increased compared with that of CXCL10 or radiotherapy alone. Flow cytometry showed that CXCL10 overexpression in HeLa cells resulted in a prolonged G1 phase and shortened $\mathrm{S}$ phase at $72 \mathrm{~h}$ post-transfection. Western blot analysis revealed that $\mathrm{p} 27^{\mathrm{Kip} 1}$ was up-regulated in CXCL10-treated HeLa cells; however, cyclin E was down-regulated. These results indicate that the combination of CXCL10 gene therapy and radiotherapy is an effective strategy for the growth suppression of HeLa cells, and that CXCL10 enhances the radiotherapy effects through cell cycle redistribution. Our data provide new insight into the treatment of cervical carcinoma, involving an effective combination of gene therapy and radiotherapy against tumors.
\end{abstract}

\section{Introduction}

Cervical cancer is the second most common cancer in women worldwide and a leading cause of cancer mortality in women in underdeveloped countries $(1,2)$. Previous studies have demonstrated that high-risk human papillomavirus (HPV) infection is a necessary factor in the development of cervical cancer (3). As early gene products of the high-risk HPV type, E6 and E7 are consistently retained and expressed in cervical carcinoma cells, and inhibit the functions of the tumor suppressors, p53 and retinoblastoma protein ( $\mathrm{pRb}$ ), through ubiquitin-dependent proteolytic degradation (4). The consistent overexpression

Correspondence to: Professor Jing-Bo Wu, Department of Oncology, Affiliated Hospital of Luzhou Medical College, Luzhou, Sichuan 646000, P.R. China

E-mail:wjb6147@163.com

Key words: CXC chemokine ligand 10, radiotherapy, gene therapy, cervical cancer, cell cycle of HPV E6 and E7 oncoproteins is required to maintain the malignant phenotype of cervical carcinoma cells, suggesting that they actively block the execution of a senescence program. Thus, these oncoproteins may be ideal targets for developing drugs against cervical cancer (5).

Radiotherapy plays a crucial role in the treatment of cervical cancer. Currently, approximately $80 \%$ of cervical cancer patients require radiotherapy $(6,7)$. Radiotherapy has been used at various clinical stages in the treatment of cervical cancer; however, its efficacy is not completely satisfactory and requires further improvement. The 5-year survival rate of stage I/II cervical cancer patients is $65-85 \%$, and that of stage III/IV patients is $20-50 \%(8,9)$. Hence, it is imperative to find alternative treatments which combine radiotherapy to form new comprehensive ways to improve the effectiveness of cervical cancer treatment.

Chemokines are a family of small cytokines secreted by cells, which participate in pleiotropic functions including cell migration, cell maturation and angiogenesis (10). Chemokines play significant roles in the biological processes of inflammation, immune surveillance and development (11). CXC chemokine ligand 10 (CXCL10)/IFN- $\gamma$-inducible protein 10 (IP-10) is known to be one of these chemokines, and is secreted by certain cell types including monocytes, endothelial cells and fibroblasts (12). The primary functions of CXCL10 are regulating the migration of monocytes/ macrophages, T cells and NK cells, eliciting potent thymusdependent anti-tumor effects in vivo (13). Conversely, CXCL10 has been shown to induce significant anti-tumor activity (14). Furthermore, CXCL10 has been reported to inhibit angiogenesis (15). These studies indicate that CXCL10 is a potent agent in the treatment of carcinoma. In the present study, we investigated the effects of CXCL10 gene therapy combined with radiotherapy on cervical cancer using HeLa cells. The study aimed to provide new insight into the treatment of cervical cancer and to explore the potential of CXCL10 in this treatment.

\section{Materials and methods}

Cell line. The cervical carcinoma cell line, HeLa, was obtained from the American Type Culture Collection (ATCC). Cells were cultured in Dulbecco's modified Eagle's medium (DMEM) supplemented with $10 \%$ fetal bovine serum (FBS), maintained in a $37^{\circ} \mathrm{C}$ incubator with a humidified $5 \% \mathrm{CO}_{2}$ atmosphere. 

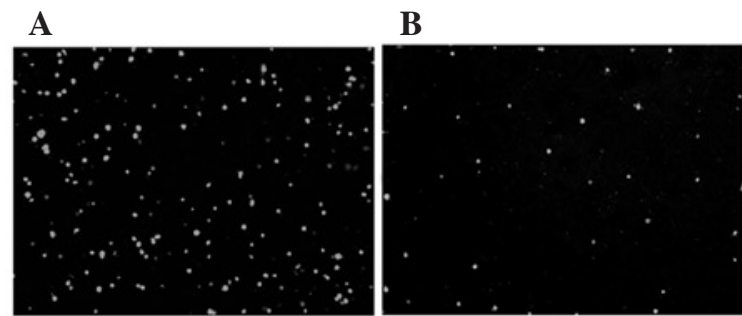

C

D
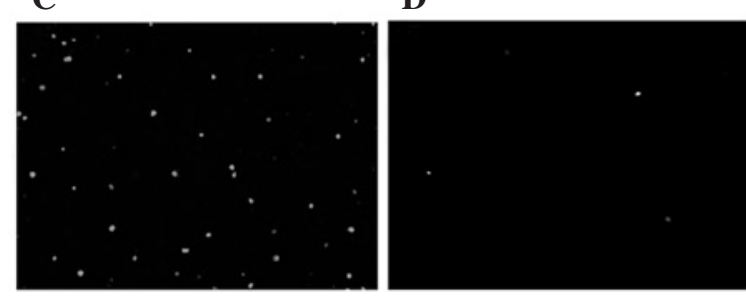

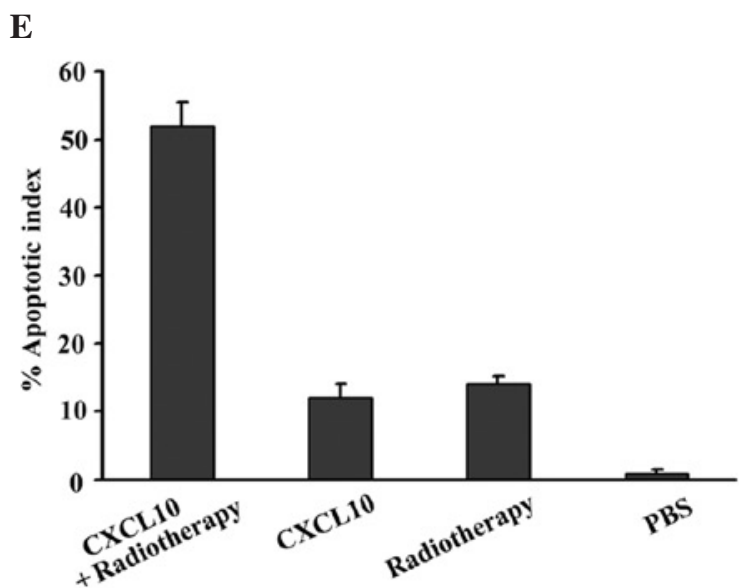

Figure 1. The combined treatment of CXCL10 gene therapy and radiotherapy increased cell apoptosis. The apoptotic cells were detected using TUNEL assay. (A) PBS, (B) CXCL10, (C) radiotherapy, (D) CXCL10 + radiotherapy, (E) quantitative measurement.

Plasmid DNA construction and preparation. The open reading frame of CXCL10 cDNA (GenBank accession: NM_001565.2) was cloned into plasmid pcDNA3.1(+) (Invitrogen, USA) between $E c o$ RI and $X h o$ I sites to obtain recombinant plasmids expressing CXCL10. As a control, the pure pcDNA3.1(+) plasmid was used as an empty vector. The plasmids were purified through two rounds of passage over EndoFree columns (Qiagen, Chatsworth, CA, USA) according to the manufacturer's instructions. The expression of plasmid DNA was confirmed in the transfected cells by using RT-PCR.

DNA transfection and radiotherapy. The cells were seeded in 96-well plates at $1 \times 10^{4}$ per well and transiently transfected with the recombinant vector of pcDNA3.1(+)-CXCL10 by lipofectamine 2000 (Invitrogen) according to the manufacturer's instructions. External beam radiotherapy using X-rays was introduced, and the seeded cells were administered a single radiation dose of 5 Gy.

Quantitative assessment of apoptosis. Cell apoptosis was determined using the DeadEnd ${ }^{\mathrm{TM}}$ fluorometric TUNEL system (Promega, Madison, USA) according to the manufacturer's instructions. The nucleus of apoptotic cells was observed under a fluorescence microscope (Axiovert 200; Carl Zeiss, Germany) at an excitation wavelength of $520 \mathrm{~nm}$. Five random fields were randomly selected and analyzed. The apoptotic index was calculated as a ratio of the apoptotic cell number to the total cell number under each field.

Flow cytometry. Cell cycle progression was analyzed using a flow cytometer (ESP Elite; Coulter, USA). Briefly, $1 \times 10^{6}$ cells were fixed in $80 \%$ cold ethanol for $30 \mathrm{~min}$ at $4^{\circ} \mathrm{C}$ and washed three times with phosphate-buffered saline (PBS). Then, the cells were incubated in the propidium iodide (PI) buffer $[50 \mathrm{mg} / \mathrm{ml} \mathrm{PI}$, $0.1 \%$ Triton X-100, $0.1 \mathrm{mM}$ EDTA(Na $)_{2}$ and $50 \mathrm{mg} / \mathrm{ml}$ RNase-A] for $30 \mathrm{~min}$ at $4^{\circ} \mathrm{C}$ in the dark until flow cytometry analysis.

Western blot analysis. Cells were dissolved in RIPA buffer on ice and centrifuged to obtain the supernatant. The extracted proteins were subjected to $12 \%$ sodium dodecyl sulfate polyacrylamide gel electrophoresis (SDS-PAGE) and transferred to PVDF membranes (Amersham Biosciences, USA). The membranes were blocked in $0.5 \%$ bovine serum albumin and incubated with primary antibodies. Then, the membranes were washed with PBST and incubated with secondary antibodies conjugated with horseradish peroxidase. After washing the membranes with PBST, the immunoblots were visualized by the enhanced chemiluminescence system SuperSignal West Pico Chemiluminescent Substrate (Pierce, USA).

\section{Results}

The combined treatment of CXCL1O and radiotherapy increases cell apoptosis. In this study, four types of treatment including PBS, CXCL10 (0.2 $\mu \mathrm{g}$ DNA), radiotherapy (5 Gy), and CXCL10 plus radiotherapy ( $0.2 \mu \mathrm{g}$ DNA and 5 Gy respectively), were applied to the cervical cancer cell line, HeLa, in vitro. TUNEL assay revealed that the cellular apoptotic rate in the CXCL10 and radiotherapy groups alone was 12 and 14\%, respectively, at $72 \mathrm{~h}$ post-treatment (Fig. 1). However, the apoptotic rate in the combined CXCL10 and radiotherapy group reached $52 \%$, approximately two-fold the sum of CXCL10 and radiotherapy alone (Fig. 1). These results indicate that CXCL10 combined with radiotherapy achieves a more positive anti-tumor effect.

Altered cell cycle induced by CXCL10 treatment. In order to establish the reason why CXCL10 increases radiotherapyinduced apoptosis, we examined the cell cycle following the treatment of HeLa cells. Flow cytometry analysis revealed that CXCL10 overexpression in HeLa cells significantly induced a prolonged G1 phase and shortened S phase at $72 \mathrm{~h}$ post-transfection, resulting in $71 \%$ of cells in the G1 phase and only $8 \%$ of cells in the S phase (Fig. 2). However, there was no obvious difference in the cell cycle following the treatment with PBS, liposome and the empty vector (Fig. 2). These data demonstrate that CXCL10 overexpression causes cell cycle redistribution. 


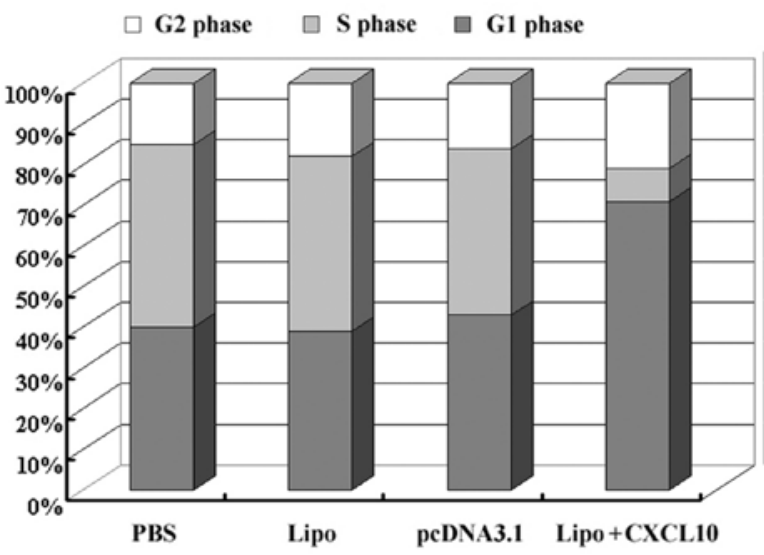

Figure 2. Altered cell cycle induced by CXCL10 treatment in HeLa cells. Flow cytometry revealed that CXCL10 overexpression in HeLa cells induced a prolonged G1 phase and shortened S phase at $72 \mathrm{~h}$ post-transfection.

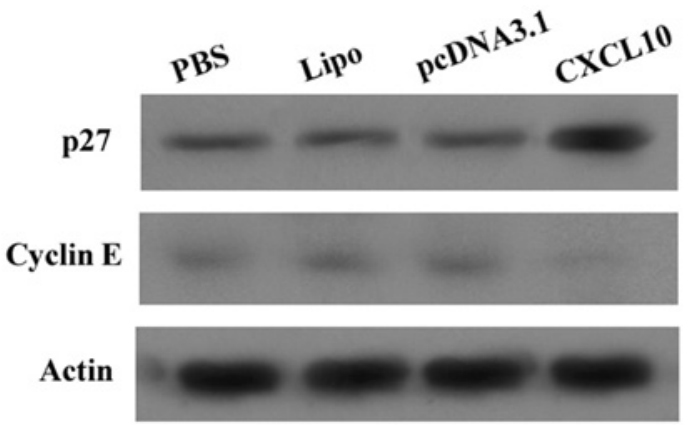

Figure 3. The changes in cell cycle proteins following CXCL10 treatment. Western blot analysis revealed that $\mathrm{p} 27^{\mathrm{Kip} 1}$ was up-regulated in CXCL10treated HeLa cells compared with the controls (PBS, liposome and the empty vector). However, cyclin E was down-regulated in CXCL10-treated HeLa cells.

Changes in cell cycle protein levels following CXCL10 treatment. To elucidate the molecular mechanism of the G1 phase arrest induced by CXCL10, the proteins involved in the cell cycle were detected by Western blot analysis. There was no obvious difference in protein levels of p27 $7^{\mathrm{Kip} 1}$ and cyclin E among cells treated with PBS, liposome and empty vector (Fig. 3). However, in CXCL10-treated cells p27 ${ }^{\mathrm{Kip1}}$ was found to be up-regulated and cyclin E down-regulated compared with the controls (PBS, liposome and the empty vector) (Fig. 3). These results indicated that the levels of cell cycle proteins were changed following CXCL10 overexpression.

\section{Discussion}

The present study was designed to examine whether the combined treatment of liposome-encapsulated CXCL10 gene therapy and radiotherapy inhibits the growth of cervical carcinoma cell line, HeLa. To our knowledge, this is the first study to demonstrate that CXCL10 gene therapy combined with radiotherapy leads to a greater inhibition of HeLa cell growth compared with CXCL10 or radiotherapy alone.

CXCL10 and radiotherapy both possess anti-cervical carcinoma efficacy; however, their anti-tumor mechanisms are different. CXCL10 induces cell apoptosis through the p53-dependent pathway initiated by the suppression of HPV
E6 and E7 expression (16). Radiotherapy induces cell apoptosis through the destruction of host genome DNA (17). We hypthesized that the combination of CXCL10 gene therapy and radiotherapy would have the following advantages, and therefore improve the efficacy of cervical carcinoma treatment. Firstly, radiation improves the efficiency of CXCL10 gene transfer into tumor cells, since the stability of the cell membrane can be weakened by radiation. Secondly, gene therapy induces cell cycle arrest in certain cases, leading to an increased sensitivity to radiation. It has been recognized that cell cycle redistribution is essential for radiation. For example, the $\mathrm{S}$ phase is least sensitive to radiation; however, gene synthesis of the $S$ phase in tumor cells is very active (18). In the present study, the markedly increased G1 and decreased S phases of HeLa cells were observed following treatment with CXCL10, which can improve the anti-tumor efficacy of radiation.

In order to reveal the molecular process of G1 arrest induced by CXCL10, two cell cycle-related proteins, cyclin E and $\mathrm{p} 27^{\mathrm{Kip} 1}$, were examined in our study. Cyclin $\mathrm{E}$ is one of the nuclear proteins and a significant regulatory factor of the cell cycle. Cyclin E binds CDK2 to form a complex that promotes cell cycle progression from the $\mathrm{G} 1$ to $\mathrm{S}$ phase and consequently induces the cell division process (19). p27 ${ }^{\mathrm{Kip} 1}$, a cyclin-dependent kinase inhibitor, plays a significant role in limiting cell cycle progression. $\mathrm{p} 27^{\mathrm{Kip} 1}$ mainly inhibits the activity of cell cycle kinase complexes such as cyclin E-CDK2, which leads to G1 phase arrest (20).p2 $7^{\mathrm{Kip} 1}$ executes its inhibitory effects in two ways. First, it inhibits the phosphorylation of CDK2-Thr160, resulting in the retarded activation process of cyclin E-CDK2. In addition, $\mathrm{p} 27^{\mathrm{Kip} 1}$ also directly binds cyclin E-CDK2 complex to inhibit the activated kinase complex (21). In this study, the decreased cyclin $\mathrm{E}$ and increased $\mathrm{p} 27^{\mathrm{Kip} 1}$ expression could explain the prolonged G1 and shortened S phase, indicating that CXCL10 causes cell cycle redistribution in HeLa cells.

In summary, we demonstrated that the combination of CXCL10 gene therapy and radiotherapy is an effective treatment strategy for the growth suppression of the cervical carcinoma cell line, HeLa. CXCL10 enhanced the radiotherapeutic effects in HeLa cells through cell cycle redistribution. Our data provides new insight into the treatment of cervical carcinoma, involving an effective combination of gene therapy and radiotherapy against tumors.

\section{References}

1. Schiffman M and Castle PE: The promise of global cervicalcancer prevention. N Engl J Med 353: 2101-2104, 2005.

2. Mandelblatt JS, Lawrence WF, Womack SM, Jacobson D, Yi B, Hwang YT, Gold K, Barter J and Shah K: Benefits and costs of using HPV testing to screen for cervical cancer. JAMA 287: 2372-2381, 2002.

3. Moody CA and Laimins LA: Human papillomavirus oncoproteins: pathways to transformation. Nat Rev Cancer 10: 550-560, 2010.

4. Schiffman M and Solomon D: Screening and prevention methods for cervical cancer. JAMA 302: 1809-1810, 2009.

5. Sørbye SW, Fismen S, Gutteberg T and Mortensen ES: Triage of women with minor cervical lesions: data suggesting a 'test and treat' approach for HPV E6/E7 mRNA testing. PLoS One 5: e12724, 2010.

6. Small W Jr, Mell LK, Anderson P, Creutzberg C, De Los Santos J, Gaffney D, Jhingran A, Portelance L, Schefter T, Iyer R, Varia M, Winter K and Mundt AJ: Consensus guidelines for delineation of clinical target volume for intensity-modulated pelvic radiotherapy in postoperative treatment of endometrial and cervical cancer. Int J Radiat Oncol Biol Phys 71: 428-434, 2008. 
7. Grigsby PW: Radiotherapy for pelvic recurrence after radical hysterectomy for cervical cancer. Radiat Med 23: 327-330, 2005.

8. Klopp AH and Eifel PJ: Chemoradiotherapy for cervical cancer in 2010. Curr Oncol Rep 13: 77-85, 2011.

9. Tanderup K, Georg D, Pötter R, Kirisits C, Grau C and Lindegaard JC: Adaptive management of cervical cancer radiotherapy. Semin Radiat Oncol 20: 121-129, 2010.

10. Bromley SK, Mempel TR and Luster AD: Orchestrating the orchestrators: chemokines in control of T cell traffic. Nat Immunol 9: 970-980, 2008.

11. Sallusto F and Baggiolini M: Chemokines and leukocyte traffic. Nat Immunol 9: 949-952, 2008.

12. Campanella GS, Colvin RA and Luster AD: CXCL10 can inhibit endothelial cell proliferation independently of CXCR3. PLoS One 5: e12700, 2010.

13. Liu M, Guo S and Stiles JK: The emerging role of CXCL10 in cancer. Oncol Lett 2: 583-589, 2011.

14. Dufour JH, Dziejman M, Liu MT, Leung JH, Lane TE and Luster AD: IFN-gamma-inducible protein 10 (IP-10; CXCL10)deficient mice reveal a role for IP-10 in effector T cell generation and trafficking. J Immunol 168: 3195-3204, 2002.

15. Zipin-Roitman A, Meshel T, Sagi-Assif O, Shalmon B, Avivi C, Pfeffer RM, Witz IP and Ben-Baruch A: CXCL10 promotes invasion-related properties in human colorectal carcinoma cells. Cancer Res 67: 3396-3405, 2007.
16. Zhang HM, Yuan J, Cheung P, Chau D, Wong BW, McManus BM and Yang D: Gamma interferon-inducible protein 10 induces HeLa cell apoptosis through a p53-dependent pathway initiated by suppression of human papillomavirus type 18 E6 and E7 expression. Mol Cell Biol 25: 6247-6258, 2005.

17. Frosina G: DNA repair and resistance of gliomas to chemotherapy and radiotherapy. Mol Cancer Res 7: 989-999, 2009.

18. Pawlik TM and Keyomarsi K: Role of cell cycle in mediating sensitivity to radiotherapy. Int J Radiat Oncol Biol Phys 59: 928-942, 2004.

19. Mazumder S, Plesca D and Almasan A: A jekyll and hyde role of cyclin $\mathrm{E}$ in the genotoxic stress response: switching from cell cycle control to apoptosis regulation. Cell Cycle 6: 1437-1442, 2007.

20. Kawauchi S, Yamamoto Y, Uchida K, Chochi Y, Kondo T, Oga A and Sasaki K: Significance of cyclin E and p27 expression in malignant ovarian germ cell tumors: correlation with the cell proliferation activity and clinicopathologic features. Oncol Rep 16: 1029-1033, 2006.

21. Bhatia B, Malik A, Fernandez A and Kenney AM: p27(Kip1), a double-edged sword in Shh-mediated medulloblastoma: Tumor accelerator and suppressor. Cell Cycle 9: 4307-4314, 2010. 